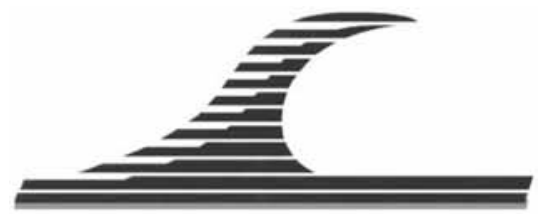

Revue Paralia, Volume 5 (2012) pp 1.1-1.14

Mots-clés : Transition solide/liquide, Erosion, Thixotropie,

Seuil d'écoulement, Sédiments cohésifs, Fluides modèles

(C) Editions Paralia CFL

\title{
Etude rhéométrique pour la mise au point de sédiments cohésifs modèles transparents pour une approche locale du comportement en érosion
}

\author{
Keang Sè POUV ${ }^{1}$, Anthony BESQ ${ }^{1}$, Sylvain GUILLOU ${ }^{1}$
}

1. Laboratoire Universitaire des Sciences Appliquées de Cherbourg, EA4253, Groupe "Mécanique des Fluides et Rhéologie", Site Universitaire, 50130 Cherbourg Octeville, France.

keang-se.pouv@unicaen.fr ; anthony.besq@unicaen.fr ; sylvain.guillou@unicaen.fr

\section{Résumé :}

Dans cette contribution, on a recourt à des essais rhéométriques pour caractériser le comportement d'un sédiment cohésif naturel dont une partie est traitée par rayonnement ionisant gamma. On montre que ce traitement conduit à une réduction importante du niveau de résistance du sédiment. Le suivi spécifique des dynamiques d'évolution de la structure du sédiment permet d'établir des relations d'échelle. Le vieillissement au repos met en évidence une loi d'évolution en puissance du temps. L'observation de la transition solide/liquide (processus similaire à l'érosion du fond sédimentaire) permet d'obtenir une relation exponentielle du temps de transition en fonction de l'inverse de la contrainte appliquée. Le fait le plus remarquable est que les dynamiques ne semblent pas affectées par le traitement par irradiation gamma. Pour mieux comprendre les phénomènes mis en jeu dans le processus d'érosion, il est proposé de suivre localement ces dynamiques à l'aide des techniques PTV et LIF. Dans cette optique, il faut disposer de fluides modèles transparents simulant les propriétés rhéologiques des sédiments naturels et notamment les dynamiques particulières présentées précédemment. Deux fluides modèles à seuil (gels) utilisant le carbopol et la laponite sont ainsi mis au point, et on montre la similitude des propriétés.

Soumis le 4 octobre 2011, accepté le 6 janvier 2012, en ligne le 23 janvier 2012.

La seule version examinée est celle écrite en français. La ou les autres versions n'étant pas examinées par le comité de rédaction de la revue, sont donc publiées sous l'entière responsabilité du ou des auteurs. 


\section{Introduction et positionnement de la problématique}

Dans les ports et les estuaires, les sédiments s'accumulent dans certaines zones et sont érodés dans d'autres. La gestion à long terme de ces espaces nécessite d'être capable de modéliser le comportement en érosion et en transport des sédiments, qui sont par ailleurs le plus souvent cohésifs. Décrire ces propriétés d'érosion requière une phase expérimentale (in situ ou en laboratoire) pour évaluer la contrainte hydrodynamique déclenchant l'érosion. Les différentes techniques et essais mis en œuvre, en soumettant le matériau à des contraintes induites par un écoulement d'eau, montrent que cette tâche est complexe. Ainsi, TOLHURST et al. (2000) rapportent que le processus d'érosion ne semble pas universel puisque deux modes d'érosion, l'un en surface et l'autre en masse, pouvant s'enchaîner, ont été observés. Une caractéristique essentielle, que l'on peut retenir de ces modes, est la manifestation du processus de rupture. Les auteurs soulignent également l'importance de l'amplitude et de la durée des incréments des sollicitations mécaniques appliquées sur le fond sédimentaire. Par ailleurs, l'identification du déclenchement du processus d'érosion suite à ces sollicitations est très délicate, et donne lieu à considérer différentes valeurs seuil, selon le degré de manifestation de l'enlèvement de matière solide (i.e. détectabilité de la quantité de matière accumulée en suspension).

Du point de vue physique, l'érosion du sédiment correspond à une transition entre un état solide et un état fluide qui nécessite d’être capable de caractériser finement la dynamique du comportement mécanique sur un domaine important de déformations. La rhéométrie peut répondre à ces exigences dans le cadre de sollicitations mécaniques en cisaillement simple obtenues en confinant le matériau entre des outils. La relative facilité de mise en œuvre permet de caractériser l'impact de différents facteurs tels que la concentration, la nature et la granulométrie des constituants minéralogiques; les composants organiques et polymériques ; ..., sur le comportement mécanique.

Les différentes études expérimentales en rhéologie des sédiments ont montré le caractère complexe et évolutif dans le temps de ces matériaux qui dépend de nombreux facteurs (concentration des particules colloïdales et grossières, contenu en matière organique, activité biologique...). Les propriétés de thixotropie et d'existence d'une contrainte seuil ont été rapportées, et une meilleure compréhension de ces propriétés a été possible, ces dernières années, grâce à une approche locale pour différents systèmes présentant les mêmes propriétés. Les travaux, ainsi conduits, mettent en évidence la coexistence de zones fluides et de zones solides au sein du même échantillon qui évoluent spatialement et temporellement selon l'état de la matière (RAYNAUD et al., 2002 ; OVARLEZ et al., 2009). Dès lors, les observations relatives à la transition solide/liquide et liquide/solide associée au concept de contrainte seuil, ainsi qu'au comportement en écoulement, sont directement dépendantes des conditions d'application des sollicitations mécaniques (distribution, temps et taux d'accroissement) (COUSSOT et al., 2002 ; UHLHERR et al., 2005). 
Cette vision de la problématique de l'érosion en tant que transition de comportement mécanique pose légitimement plusieurs questions. Compte tenu de la différence de la nature de la sollicitation mécanique en érosion et en rhéométrie, est-il possible de trouver un lien permettant de prévoir le comportement en érosion à partir de "simples" essais rhéométriques ? Une étude spécifique peut être menée dans ce sens, mais elle impose d'une part, d'éliminer l'évolutivité du sédiment inhérente à la composante biologique, et d'autre part, d'obtenir simultanément des informations macroscopiques caractérisant les dynamiques et des informations locales du matériau qui adoptera vraisemblablement une organisation hétérogène. Quelles techniques peut-on alors mettre en œuvre?

Les techniques "classiques" de RMN, d'imagerie de particules (PIV ou PTV), de vélocimétrie Doppler (ondes sonores ou laser) peuvent répondre à ces objectifs. Elles se différencient par leur caractère potentiellement intrusif (ajout ou non de traceurs), leur résolution spatiale et temporelle ainsi que leur exigence de certaines propriétés du fluide (transparence optique, homogénéité). La technique exploitant la fluorescence induite par laser (LIF) peut également apporter des informations qualitatives sur les phénomènes de diffusion et de déformation au sein du matériau. La conséquence immédiate du choix de techniques basées sur l'imagerie optique est la nécessité d'utiliser un matériau modèle transparent. Cette contrainte offre la possibilité de développer un matériau avec des propriétés ajustables, maîtrisées et stables dans le temps. Il reste alors à définir la nature des propriétés que l'on souhaite contrôler.

Dans cet article, on aborde la problématique de l'érosion de sédiments cohésifs en étudiant les propriétés rhéologiques fondamentales de contrainte seuil et de thixotropie d'un sédiment naturel, en exploitant plutôt des essais de fluage pour caractériser les aspects dynamiques qui ne peuvent être identifiés précisément avec les essais classiques d'écoulement. Ce sédiment sera par ailleurs traité de manière à le perturber et souligner l'importance de la composante biologique. Les résultats obtenus constituent une référence pour la mise au point de matériaux modèles. Ce sont ceux déjà présentés par POUV et al. (2010). On décrit, par ailleurs, le protocole de préparation de deux fluides modèles transparents et leur caractérisation démontre la similitude de comportement avec celui des sédiments cohésifs naturels. Ces deux fluides modèles (un gel de carbopol et un gel mixte laponite-carbopol) possèdent un même niveau de seuil et se distinguent par leur caractère thixotrope qui est une composante souvent peu prise en compte dans les études d'érosion. On rappelle que le carbopol (polymère) et la laponite (argile de synthèse de type hectorite) sont des additifs très utilisés et étudiés en rhéologie. Une description détaillée de ces constituants de base est faite par PIAU (2007) et MONGONDRY (2003). 


\section{Méthodologie}

\subsection{Echantillons et caractéristiques d'un sédiment naturel}

L'échantillon de sédiments marins a été collecté en février 2008, à la benne dans la grande rade de Cherbourg (Lat. 4940'05.00"N, Long. 01³6'11.00"O) à proximité de la voie maritime de la Passe de l'Est. L’échantillon a été réparti équitablement dans quatre tonnelets hermétiques de $6 \mathrm{l}$ qui ont été transférés le jour même vers le site de Sablé sur Sarthe (72), de la société Ionisos. Un traitement par irradiation gamma (dose de 35 KGy en périphérie) a alors été pratiqué sur seulement 2 colis sans ouverture de ces derniers. Le temps total entre l'envoi et le retour des tonnelets au laboratoire a été de 3 jours. Ce traitement initial permet ainsi d'obtenir, pour des mêmes conditions de stockage, un échantillon caractérisé par une activité biologique normale (croissance/décroissance de bactéries, production de substances extracellulaires, ...) et un autre où à la fois la population bactérienne et les substances polymériques ont été endommagées voir détruites. Ces échantillons seront qualifiés par la suite de naturels et d'irradiés.

La dernière étape de préparation consiste en un tamisage étagé, effectué en trois passes à l'aide de tamis normalisés, avec une taille ultime de coupure de $125 \mu \mathrm{m}$.

On note une valeur du pH des sédiments, qu'ils soient irradiés ou non, voisine de 7,5. La concentration massique en matière solide des échantillons a été déterminée à partir de trois prélèvements pour chaque type de traitement (naturel, irradié), en considérant les valeurs de pesée avant et après séchage à l'étuve (24 h à $\left.70^{\circ} \mathrm{C}\right)$. On obtient respectivement pour les systèmes naturels et irradiés, une valeur de $36,1 \pm 1,7 \%$ et de $36,0 \pm 0,1 \%$. En moyenne, les systèmes sont de même concentration. On note, cependant, une plus grande variabilité des valeurs pour le système naturel.

La densité du sédiment sec des mêmes échantillons a été mesurée par pycnométrie à hélium (Accupyc1330, Micromeritics). Une faible variabilité (0,3\%) est constatée pour l'ensemble des échantillons qui présentent une densité moyenne de 2,58g/cm³.

Ces analyses permettent de juger de la bonne homogénéité du matériau quel que soit l'échantillon examiné. Aucunes autres analyses n’ont été faites car l'objectif des essais rhéométriques n’est pas d'établir de corrélation entre le comportement mécanique et la composition du matériau.

\subsection{Préparation des sédiments modèles}

La fabrication des gels de carbopol commence par le mélange d'une masse appropriée de carbopol (0,3\%) (Ultrez 21, Noveon) dans de l'eau ultra-pure (14,45\%) (Chromasolv 34877, Sigma Aldrich), à l'aide d'un agitateur magnétique (500 tr/min) pendant $20 \mathrm{~min}$. La quantité correspondante de glycérol (85\%) (24397.236, VWR International) est ensuite ajoutée dans le mélange et agitée à $1100 \mathrm{tr} / \mathrm{min}$ pendant $20 \mathrm{~min}$. Puis, un temps de repos de 20 min est respecté pour que les particules de carbopol puissent bien s'hydrater et gonfler librement dans la solution. La dernière étape consiste à neutraliser 
le système par ajout de Triéthanolamine (0,25\%) (Fluka 90280, Sigma Aldrich). Au cours de la neutralisation, le mélange est agité manuellement à l'aide d'une spatule métallique jusqu'à l'obtention d'un gel homogène (20 à $30 \mathrm{~min}$ ). Il faut noter que les pourcentages indiqués représentent les concentrations en poids de chaque constituant.

Après une durée d'environ $24 \mathrm{~h}$, les valeurs du $\mathrm{pH}$ et de la conductivité du gel sont respectivement aux alentours de 7,13 et de 4,90 $\mu \mathrm{s} / \mathrm{cm}$. Afin de limiter l'effet du vieillissement et d'obtenir une bonne reproductibilité de préparation, le gel fabriqué est mélangé à l'aide d'un homogénéisateur (Ultra-turrax) à $11000 \mathrm{tr} / \mathrm{min}$ pendant $2 \mathrm{~min}$, puis réparti dans des tubes pour une opération de centrifugation à $5000 \mathrm{tr} / \mathrm{min}$ pendant 20 min afin d'éliminer toutes les bulles d'air existantes. Dès lors, on obtient un gel transparent d'indice de réfraction à $17^{\circ} \mathrm{C}$ (mesuré à l'aide d'un réfractomètre Euromex) de 1,446 et de masse volumique de $1,223 \mathrm{~g} / \mathrm{cm}^{3}$.

Les gels mixtes laponite-carbopol sont obtenus par mélangeage de 45,9\% d'une suspension de laponite à $1 \%$ (âgé de 2 jours), 39,1\% d'un gel de carbopol à 0,3\% (âgé de 2 jours) et 15\% de glycérol. La préparation des gels de carbopol s'effectue selon la méthode décrite auparavant (sans l'agitation finale avec l'Ultra-turrax). Le protocole de réalisation des dispersions de laponite à $1 \%$ est divisé en deux étapes se déroulant sur deux jours consécutifs. Deux mélanges de même masse sont réalisés le premier jour : l'un est la dissolution directe de $\mathrm{NaCl}$ (S/3160/53, Fisher Scientific) à $2.10^{-3} \mathrm{~mol} / \mathrm{l}$ dans de l'eau ultra-pure, et l'autre est la dispersion de poudre de laponite (RD, Rockwood Additives) à 2\% dans de l'eau ultra-pure à l'aide de l'Ultra-turrax (11000 tr/min pendant 15 min). Le jour suivant, les deux solutions sont mélangées entièrement au moyen de l'Ultra-turrax (11000 tr/min pendant $15 \mathrm{~min}$ ). On obtient ainsi une suspension de laponite ayant une concentration massique de $1 \%$ et une force ionique de $10^{-3} \mathrm{~mol} / \mathrm{l}$. Le mélange entre le glycérol, les suspensions de laponite et de carbopol s'effectue vigoureusement à l'aide de l'Ultra-turrax (11000 tr/min pendant $2 \mathrm{~min})$. On réalise, pour finir, une étape de centrifugation (5000 tr/min pendant $3 \mathrm{~min}$ ), en tubes pour éliminer complètement les bulles d'air. On obtient ainsi un gel transparent d'indice de réfraction de 1,392 et de masse volumique de $1,153 \mathrm{~g} / \mathrm{cm}^{3}$.

\subsection{Techniques rhéométriques}

Les essais rhéométriques ont été effectués avec deux rhéomètres à contrainte imposée : AR1000 (TA Instruments) utilisé avec une géométrie plan-plan de $40 \mathrm{~mm}$ de diamètre pour les sédiments naturels, et Rhéoscope1 (Thermo Fisher Scientific) utilisé avec une géométrie plan-plan de $28 \mathrm{~mm}$ de diamètre pour les sédiments modèles. La surface des deux géométries est couverte de dents pyramidales (base 1x1mm², hauteur $867 \mu \mathrm{m}$ ) directement usinées dans la matière. Ces géométries cloutées s'apparentent à celle développée par NICKERSON \& KORNFIELD (2005) afin de s’affranchir des problèmes de glissement. Des études complémentaires ont montré que ce type de 
géométrie permettait également de maîtriser l’homogénéité du champ de cisaillement sur un domaine beaucoup plus grand de valeurs de contrainte (POUV et al., 2009).

Un entrefer de $2 \mathrm{~mm}$ est utilisé pour la géométrie dentée PP40, ce qui est suffisant en regard de la taille des particules présentes dans les échantillons de sédiment naturel. Cependant, un entrefer de $1 \mathrm{~mm}$ est utilisé pour la géométrie dentée PP28. Compte tenu de la taille des dents, l'épaisseur de cisaillement effective est plus grande que l'entrefer. La quantité supplémentaire à considérer pour pouvoir calculer les grandeurs rhéométriques s'obtient en suivant la méthode d'étalonnage proposée par NICKERSON \& KORNFIELD (2005). Dans notre cas, il faut considérer respectivement les valeurs de $165 \mu$ m et de $108 \mu$ m pour chaque plan des géométries PP40 et PP28.

\section{Résultats de l'étude rhéométrique}

\subsection{Comportement en écoulement}

Les essais d'écoulement examinés dans ce point consistent à mettre le matériau naturel, dans un premier temps, dans un état relatif dépendant du protocole retenu (précisaillement à $100 \mathrm{~s}^{-1}$ pendant $300 \mathrm{~s}$ puis repos de $300 \mathrm{~s}$ ) compte tenu du caractère thixotrope, puis à imposer une série de sollicitation par valeurs croissantes puis décroissantes (durée totale $40 \mathrm{~min}$ ). On impose soit la contrainte (CI) ou la vitesse de cisaillement (VI) via un asservissement de la contrainte. Les courbes d'écoulement (figure 1) obtenues pour ces deux modes et les deux types de sédiment mettent clairement en évidence que le système irradié est mécaniquement moins résistant, puisque les niveaux de contrainte mis en jeu pour obtenir un écoulement sur la même gamme de vitesse de cisaillement sont systématiquement plus petits. Ce constat de biostabilisation mécanique du sédiment est cohérent avec la description des effets biologiques présentés dans l'ouvrage de WINTERWERP \& VAN KESTEREN (2004). En mode contrainte imposée, les courbes présentent une première phase où la vitesse de cisaillement évolue peu. Ce régime traduit le caractère solide du sédiment qui se déforme élastiquement sous l'effet de l'accroissement de la contrainte. Au-delà de la valeur de contrainte critique (52 Pa pour le sédiment naturel et 38 Pa pour le sédiment irradié), une transition brutale s’opère et se matérialise par un quasi-plateau en contrainte, qui correspond en fait à un écoulement instable. Il a été montré que la stabilité n'était possible qu'au-delà d'une vitesse de cisaillement critique pour laquelle l'état du fluide est homogène au sein de l'entrefer. Il est important de noter que la transition solide/liquide est associée à une dynamique propre ce qui fait que la contrainte critique correspond à un changement observable sur l'échelle de temps défini par l'essai. Cette valeur est donc plus grande que la valeur de contrainte seuil qui permet d'initier la transition. A la fin du plateau, on est en présence d'un écoulement homogène associé à une vitesse de cisaillement compatible avec le niveau de contrainte imposé. On assiste ensuite à une augmentation conjointe de la vitesse de cisaillement avec la 
contrainte qui traduit une déstructuration progressive du sédiment. Cet état déstructuré perdure lorsque les valeurs de contrainte diminuent, si bien que la courbe de descente se trouve en dessous de la courbe de montée. Lorsque le système atteint la valeur de vitesse de cisaillement critique $\left(10 \mathrm{~s}^{-1}\right.$ pour le sédiment naturel et $30 \mathrm{~s}^{-1}$ pour le sédiment irradié), une bifurcation vers l'état solide a lieu, les vitesses de cisaillement diminuent alors rapidement.

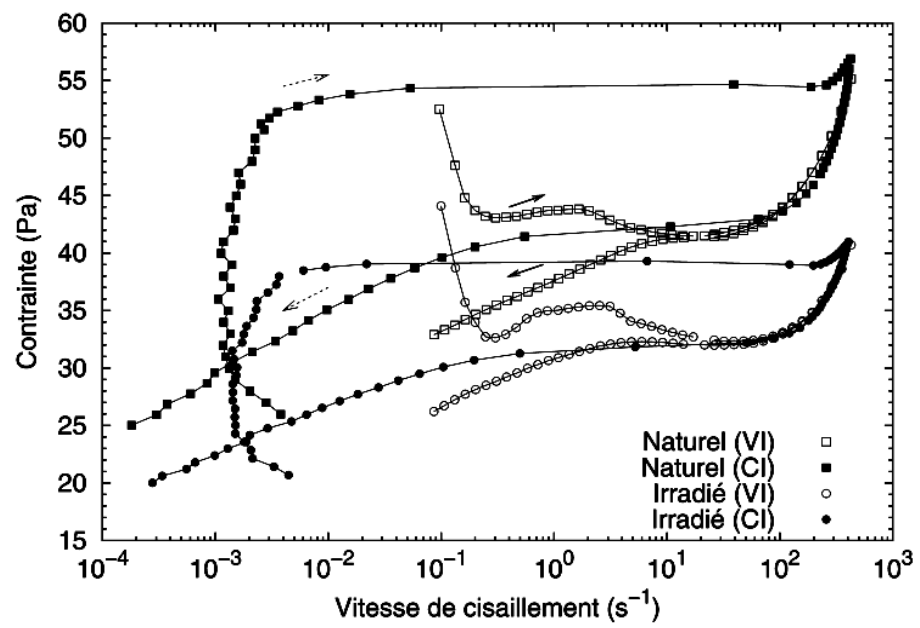

Figure 1. Courbes d'écoulement pour le sédiment naturel (carré) et irradié (cercle) en fonction du mode d'imposition des sollicitations mécaniques. Les flèches indiquent la charge et décharge du système.

Les courbes obtenues pour les essais réalisés en imposant la vitesse de cisaillement, bien que différentes des précédentes, sont parfaitement cohérentes. Lors de la montée, on assiste à une évolution non monotone tant que l'écoulement est instable (coexistence de zones solides et cisaillées), c'est-à-dire pour des vitesses de cisaillement inférieures à la vitesse de cisaillement critique. Lorsque l'on est en présence d'un écoulement stable où le matériau est homogène et déstructuré dans tout l'entrefer, la courbe de descente se superpose sur celle enregistrée lors des essais en mode contrainte imposée. Dans cette zone, on constate peu de différences entre la courbe de montée et de descente ce qui suggère que, à partir du moment où tout l'entrefer est cisaillé de manière homogène, la cinétique de déstructuration mise en jeu est plutôt lente. Enfin, pour les courbes de descente, on retrouve le phénomène de bifurcation dès lors que la vitesse de cisaillement est inférieure à la valeur critique. Les courbes se distinguent de celles obtenues dans le cas des essais en mode contrainte imposée car les conditions d'application de la sollicitation donnent lieu à une distribution différente des contraintes au sein de l'échantillon.

Pour les matériaux modèles, seuls les essais d'écoulement en mode CI (précisaillement de $\pm 1 \mathrm{~s}^{-1}$ pendant $90 \mathrm{~s}$, temps de repos de $120 \mathrm{~s}$, et durée totale de l'application de 
contrainte de $60 \mathrm{~min}$ ) sont effectués. Pour ces conditions d'essai, on obtient des comportements similaires à ceux rencontrés précédemment pour les sédiments naturels. Les courbes d'écoulement illustrées sur la figure 2 montrent que l'on est en présence de deux matériaux qui présentent une même réponse de type solide élastique. On peut estimer que les valeurs de contrainte seuil sont voisines de $16 \mathrm{~Pa}$. Le passage à l'écoulement s'effectue très progressivement pour le gel de carbopol et beaucoup plus brutalement pour le gel mixte carbopol-laponite comme c’était le cas pour les sédiments naturels. La valeur de la vitesse de cisaillement critique du gel de carbopol (environ $0,01 \mathrm{~s}^{-1}$ ) est ainsi beaucoup plus faible que celle du gel mixte laponite-carbopol (environ $2 \mathrm{~s}^{-1}$ ). La différence entre les courbes de montée et de descente suggère que seul le gel mixte laponite-carbopol possède un caractère thixotrope plus prononcé. C'est le système qui s’apparente le plus au sédiment naturel traité dans cette étude.

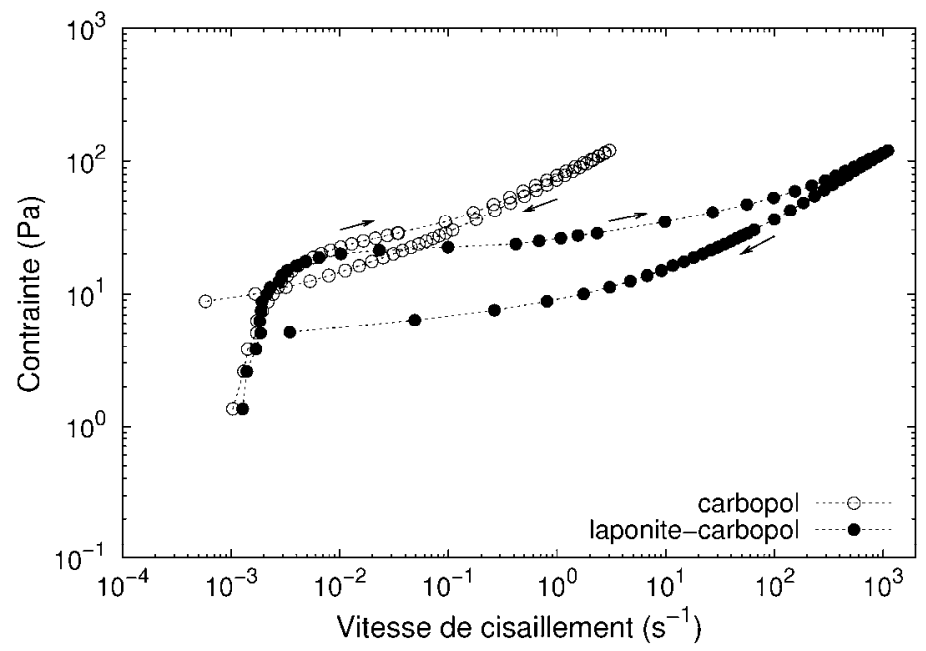

Figure 2. Courbes d'écoulement des sédiments modèles obtenues avec le mode de contrainte imposée. Les flèches indiquent la charge et décharge du système.

\subsection{Dynamique de vieillissement}

Pour évaluer le niveau de résistance du sédiment atteint après une période de repos $t w$ faisant suite à remaniement contrôlé (300 s de précisaillement à $100 \mathrm{~s}^{-1}$ qui est une valeur plus grande que la vitesse de cisaillement critique), on réalise des essais de fluage avec une même valeur de contrainte de 40,8 Pa pour le sédiment naturel et 28,8 Pa pour le sédiment irradié. Ces valeurs sont volontairement inférieures aux valeurs de contrainte critique de manière à s'approcher au mieux de la valeur de contrainte seuil que l'on ne cherche pas à déterminer précisément. Quatre valeurs du temps de repos tw ont été retenues (300, 900, 1800 et 3600 s). Ce temps de repos est classiquement vu comme un temps de vieillissement du matériau au cours duquel une réorganisation structurelle se déroule. C'est pourquoi, on choisit de prendre comme origine des temps l'instant faisant suite au précisaillement. PHAM VAN BANG et al. (2007) ont observé 
que cette réorganisation conduisait à une augmentation de la contrainte seuil avec le temps de repos; dès lors les résultats d'une étude d'érosion deviennent directement dépendants de l'histoire mécanique du sédiment (sollicitations et repos). Il est important de souligner que ce vieillissement, traduisant une reprise de structure du matériau, ne doit pas être confondu avec une évolution des propriétés au cours du stockage due à la composante biologique et organique. Les deux phénomènes existent mais avec des échelles de temps différentes.

La figure 3 présente l'évolution de la déviation angulaire (i.e. la déformation) de la géométrie au cours du temps pour le sédiment naturel. Une même réponse linéaire est enregistrée dans les tous premiers instants. Elle traduit un comportement de type solide élastique. On observe ensuite un régime oscillatoire qui correspond au couplage entre la réponse viscoélastique du matériau et l'inertie du rhéomètre. L'évolution devient par la suite continue et on assiste à une hiérarchisation des niveaux de déformation selon le temps de repos. Pour finir, on constate pour les temps longs une transition complexe vers un régime d'écoulement mise en évidence par l'incurvation des courbes. La transition solide/liquide s'amorce d'autant plus tardivement que le temps de repos est grand.

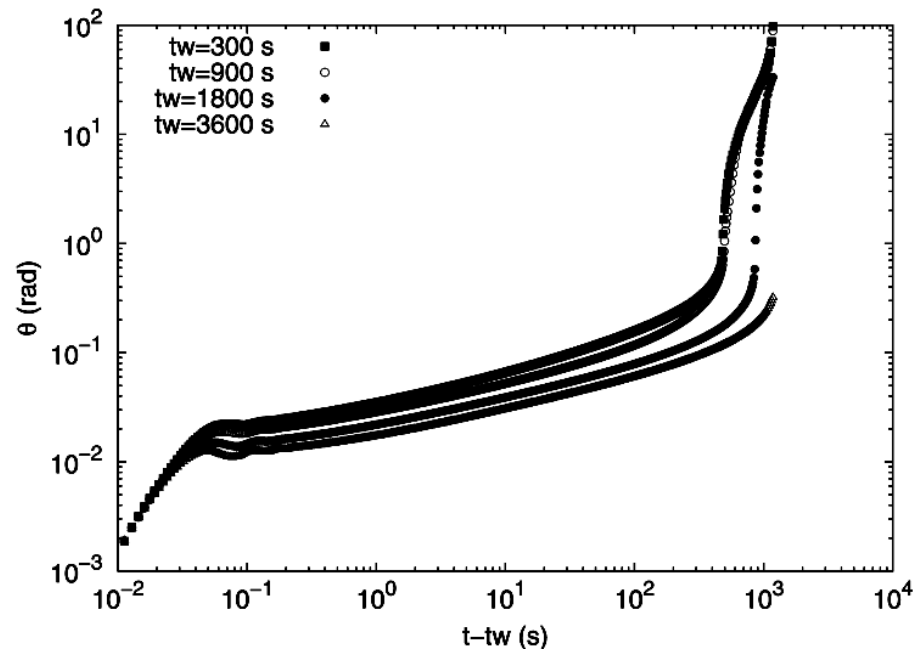

Figure 3. Courbes de fluage à 40,8 Pa pour le sédiment naturel en fonction du temps et pour différents temps de repos tw.

Pour caractériser la dynamique de vieillissement, on utilise ces essais pour relever le temps nécessaire afin d'atteindre un même niveau de complaisance J0 (i.e. même rapport déformation sur contrainte). Le tracé en double échelle logarithmique des valeurs de temps pour $J 0=1,08.10^{-2} \mathrm{~Pa}^{-1}$ en fonction du temps de repos, pour les sédiments naturels et irradiés, suggère un même comportement en loi de puissance d'exposant $\mu$ valant 1,43 (figure 4.a). La valeur de $\mu$ ne dépend pas de la valeur $J 0$ fixée. 
L’origine physique du processus de vieillissement reste à déterminer. En tout cas, il ne semble pas affecté par la présence des substances polymériques qui doivent avoir un poids moléculaire plus faible dans le cas du sédiment irradié.

L'utilisation de cette loi de puissance permet alors d'adimensionner la variable temps et d'obtenir des courbes de fluage selon une même courbe maîtresse (figure 4.b). Les courbes restent toutefois différentes pour la partie caractérisée par la dynamique de transition solide/liquide qui dépend du réseau structurel développé au sein de l'échantillon.
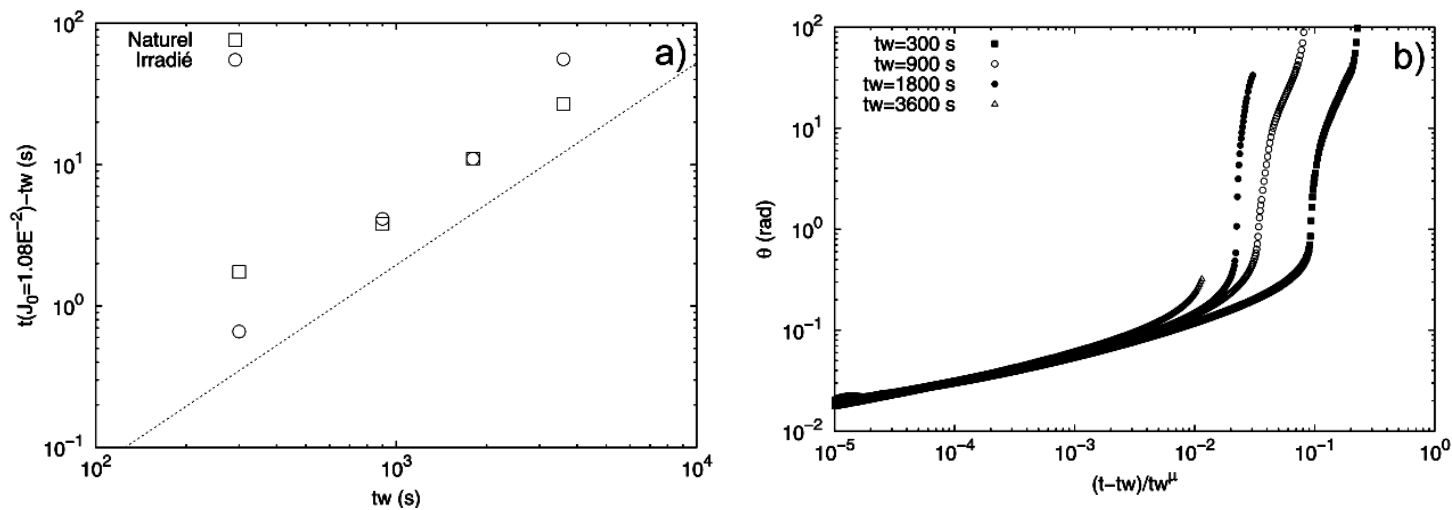

Figure 4. a. Evolution du temps d'atteinte d'une même valeur de complaisance pour les sédiments naturel et irradié (la droite matérialise une pente de 1,43). b. Utilisation de la loi de vieillissement pour adimensionner les courbes de fluage.

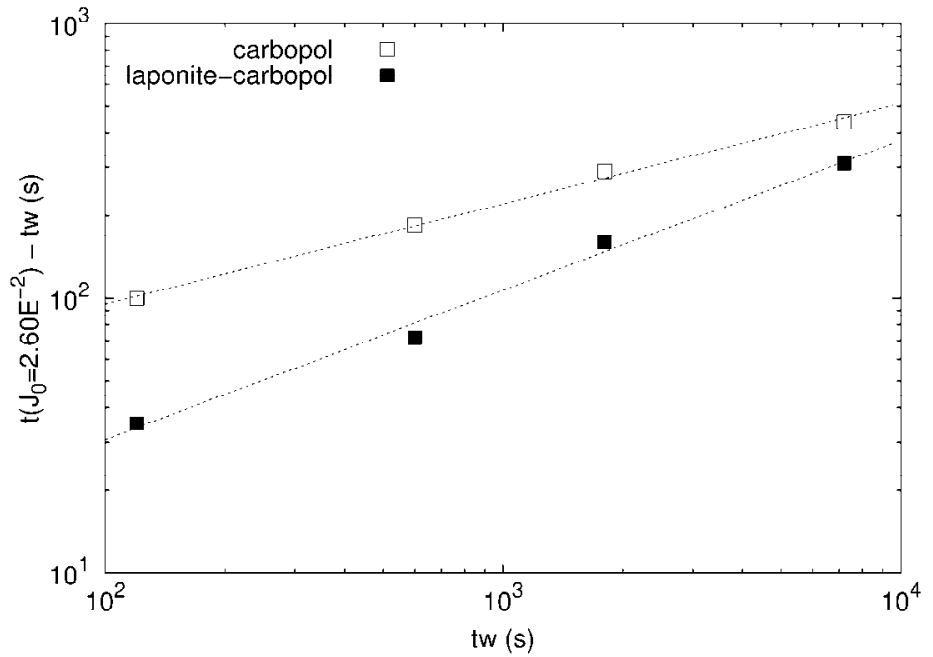

Figure 5. Valeurs de temps pour $\mathrm{JO}=2,60.10^{-2} \mathrm{~Pa}^{-1}$ en fonction du temps de repos (cas de sédiments modèles). Les deux droites matérialisent une pente de 0,40 pour le gel de carbopol et une pente de 0,55 pour le gel mixte laponite-carbopol. 
La figure 5 met en évidence qu’il existe également les phénomènes de vieillissement pour les deux sédiments modèles. Les exposants des lois de puissance obtenus (0,40 et 0,55 respectivement pour les gels de carbopol et de laponite-carbopol) sont cohérents avec les résultats trouvés dans la littérature $(0,91$ pour le gel de carbopol concentré à 1,8\% (TABUTEAU, 2005), entre 0,5 et 0,7 pour les suspensions colloïdales concentrées (DEREC et al., 2000)). L'exposant de vieillissement du gel de carbopol est plus petit que celui du gel mixte laponite-carbopol car son caractère thixotrope (niveau de restructuration dépendant du temps) est beaucoup moins prononcé. Cela diffère du cas des sédiments naturels (traités ou non) où la résistance mécanique n’est pas la même mais le caractère thixotrope semble identique (mêmes temps caractéristiques). Ces réponses soulignent l'importance de caractériser le comportement mécanique du matériau à la fois du point de vue thixotropie et seuil de contrainte.

\subsection{Dynamique de la transition solide-liquide}

Partant d'un même état structurel initial obtenu en conditionnant l'échantillon de sédiment au moyen d'une phase de précisaillement ( 5 min à $\left.100 \mathrm{~s}^{-1}\right)$ suivi d'un repos de 5 min, on examine de nouveau le comportement lors d'essais de fluage pour différentes valeurs de contrainte situées au voisinage de la contrainte seuil. On analyse ces essais en examinant l'évolution temporelle du taux de déformation. Pour le sédiment naturel, la figure 6.a présente les réponses enregistrées une fois la phase oscillatoire vue précédemment achevée. On assiste à une première étape de diminution en loi de puissance du temps qui est suivie, pour peu que la contrainte soit suffisante et que le temps d'observation soit assez grand, par un minimum temporel correspondant à un changement qualitatif du comportement du matériau. L'abscisse temporelle de ce minimum augmente lorsque la contrainte diminue. La dernière étape qui fait suite à ce minimum peut amener le matériau à l'écoulement.

Ce minimum est la seule quantité objective mesurable déjà rapportée par CATON \& BARAVIAN (2007) pour d'autres fluides et qui a été appelée temps de transition. Cela dit, il reste à connaître l'organisation locale qu'adopte le matériau au sein de l'entrefer jusqu'à ce que ce temps soit atteint. Le graphique en échelle semi-logarithmique donnant l'évolution du temps de transition en fonction de l'inverse de la contrainte $\tau$ appliquée (figure 6.b) présente une même évolution selon une loi exponentielle de la forme a.exp $(b / \tau)$ pour les sédiments naturel et irradié. Ce qui est étonnant c'est que les deux matériaux semblent être caractérisés par une même valeur de $b$. La valeur de $a$ diffère car, les matériaux ne présentant pas le même niveau de résistance mécanique, il est logique d'obtenir des temps de transition d'autant plus courts que le niveau de résistance est faible.

Le même principe d'essai (précisaillement de $\pm 1 \mathrm{~s}^{-1}$ pendant $90 \mathrm{~s}$, et temps de repos de 120 s) est opéré pour les sédiments modèles. Les évolutions exponentielles du temps de transition en fonction de l'inverse de contrainte obtenues avec les deux sédiments 
modèles sont analogues et caractérisées par la même pente $b$ mais différents coefficients $a$ associés au niveau de résistance mécanique de chaque matériau (figure 7). Il n’est pas étonnant que les valeurs de $a$ et $b$ obtenues dans les cas de sédiments naturels et modèles soient différentes car ces deux familles de matériau ne présentent pas des organisations structurelles identiques et des interactions entre les constituants de même nature.
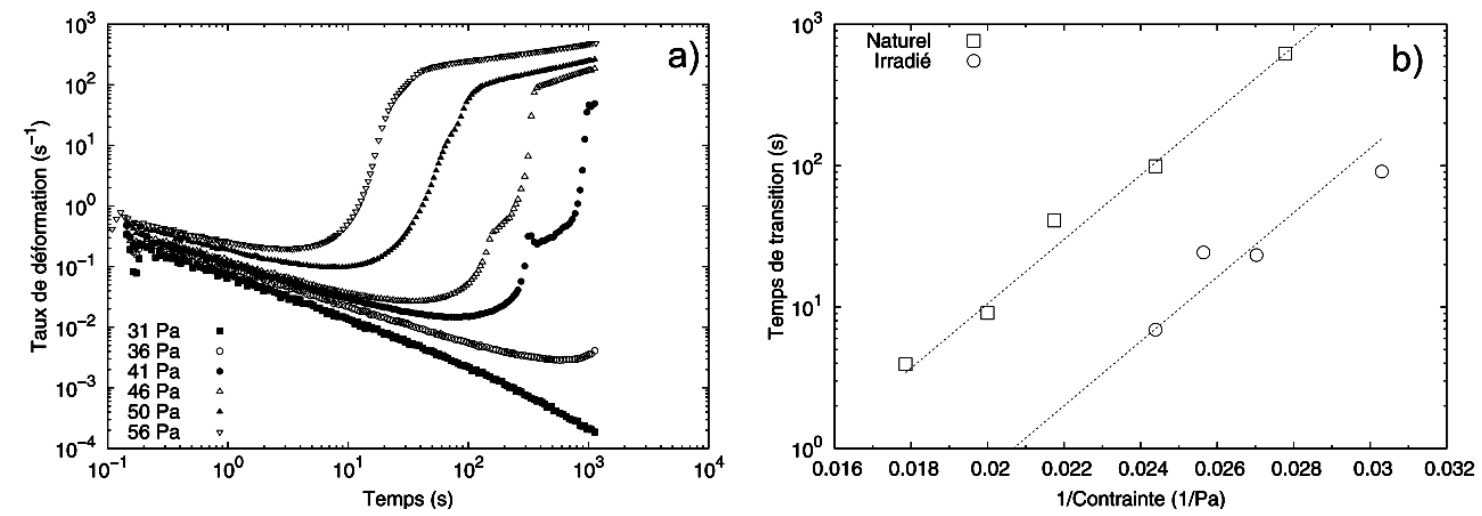

Figure 6. a. Evolution du taux de déformation calculé à partir des essais de fluage (cas du sédiment naturel). $b$. Evolution de la position temporelle du minimum du taux de déformation pour les sédiments naturel et irradié.

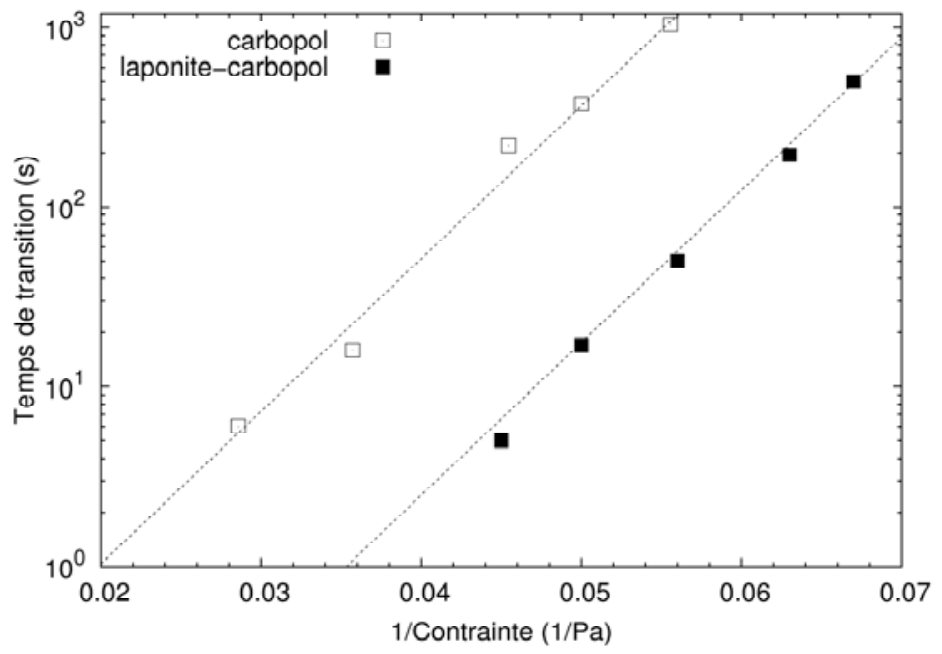

Figure 7. Evolution de la position temporelle du minimum du taux de déformation pour les sédiments modèles.

Ces résultats soulignent donc l'importance de l'échelle de temps d'observation dans le processus de transition solide/liquide du fait des propriétés dépendantes du temps (degré de seuil et de thixotropie). Cette échelle de temps dépend intimement des conditions expérimentales (niveau de contrainte, état initiale). Cela confirme l'intérêt d'être en 
Etude rhéométrique pour la mise au point de sédiments cohésifs modèles transparents pour une approche locale du comportement en érosion : 1.13

mesure de caractériser la dynamique de déformation/rupture lors d'essais d'érosion plutôt que d'observer un enlèvement de matière.

\section{Conclusion}

Les essais d'écoulement sont des essais simples de mise en œuvre qui permettent d'obtenir, pour peu que l'on exploite un domaine de valeurs de vitesse de cisaillement suffisamment grand, une première description d'un matériau. Les essais réalisés ont montré que les sédiments étudiés étaient des fluides à seuil thixotropes et qu'un traitement par irradiation gamma conduit à une réduction importante du niveau de résistance du sédiment, du fait de l'endommagement des éléments stabilisants inhérents à la présence de microorganismes. Des essais complémentaires en fluage ont été réalisés pour suivre les dynamiques d'évolution de la structure du sédiment au cours du vieillissement et de la transition solide/liquide. Une loi d'évolution en puissance du temps et exponentielle en inverse de la contrainte appliquée a respectivement été identifiée pour ces deux cas. Le fait le plus remarquable est que les dynamiques ne semblent pas affectées par le traitement par irradiation gamma.

Sur la base de ces résultats, un travail de mise au point de fluides modèles transparents a été entrepris et a débouché sur une méthode de préparation de deux fluides modèles à partir d'eau, de glycérol, de carbopol (polymère) et de laponite (argile de synthèse de type hectorite). Les premières caractérisations montrent que l'on est en présence de deux fluides présentant des valeurs de contrainte seuil voisines et des caractères thixotropes différents. Il a été mis en évidence que les comportements de ces fluides modèles et des sédiments naturels étudiés étaient similaires. La caractérisation des dynamiques locales des fluides modèles fera l'objet d'une étude ultérieure. Cette dernière sera menée à partir de suivis particulaires de traceurs pour deux modes de sollicitation mécanique, l'un en érosion, l'autre en rhéométrie. Compte tenu des résultats présentés, un intérêt particulier sera porté à la manière dont un saut en contrainte est pratiqué, de manière graduelle ou instantanée.

\section{Références bibliographiques}

CATON F., BARAVIAN C. (2007). Du vieillissement à la rupture des fluides à seuils. 42ème colloque du Groupe Français de Rhéologie, Clermont-Ferrand.

COUSSOT P., NGUYEN Q.D., HUYNH H.T., BONN D. (2002). Viscosity bifurcation in thixotropic, yielding fluids. Journal of Rheology, 46(3), pp 573-589. doi:10.1122/1.1459447

DEREC C., AJDARI A., DUCOURET J., LEQUEUX F. (2000). Rheological characterization of aging in a concentrated colloidal suspension. C. R. Acad. Sci. Paris, t. 1, Série IV, pp 1115-1119. doi:10.1016/S1296-2147(00)01106-9

MONGONDRY P. (2003). Structure et comportement rhéologique des suspensions aqueuses de Laponite en présence de plusieurs additifs. Thèse, Université du Maine 
NICKERSON C.S., KORNFIELD J.A. (2005). A "cleat" geometry for suppressing wall slip, Journal of Rheology, 49(4), pp 865-874. doi:10.1122/1.1917846

OVARLEZ G., RODTS S., CHATEAU X., COUSSOT P. (2009). Phenomenology and physical origin of shear localization and shear banding in complex fluids. Rheologica Acta, 48(8), pp 831-844. doi:10.1007/s00397-008-0344-6

PHAM VAN BANG D., OVARLEZ G., TOCQUER L. (2007). Effets de la densité et de la structuration sur les caractéristiques rhéologique de la vase. La Houille Blanche, 2, pp 85-93. doi:10.1051/lhb:2007023

PIAU J.M. (2007). Carbopol gels: Elastoviscoplastic and slippery glasses made of individual swollen sponges meso- and macroscopic properties, constitutive equations and scaling laws. Journal of Non-Newtonian Fluid Mechanics, 144, pp 1-29. doi:10.1016/j.jnnfm.2007.02.011

POUV K.S., BESQ A., GUILLOU S. (2009). Outils rhéo-optiques pour la caractérisation macroscopique et locale de matériaux transparents. 44ème colloque du Groupe Français de Rhéologie, Strasbourg.

POUV K.S., BESQ A., GUILLOU S. (2010). Caractérisation rhéométrique des conditions de transition solide/liquide de sédiments cohésifs : vers un lien avec le comportement en érosion ? Journées Nationales Génie Côtier - Génie Civil, Les Sables d'Olonne, pp 529-538. doi:10.5150/jngcgc.2010.062-P

RAYNAUD J.S., MOUCHERONT P., BAUDEZ J.C., BERTRAND F., GUILBAUD J.P., COUSSOT P. (2002). Direct determination by NMR of the thixotropic and yielding behavior of suspensions. Journal of Rheology, 46(3), pp 709-732. doi:10.1122/1.1463420

TABUTEAU H. (2005). Etude du comportement de fluides viscoélastiques sur un disque en rotation. Applications à l'épandage des boues résiduaires. Thèse, Ecole Nationale du Génie Rural des Eaux et des Forêts, 152 p.

TOLHURST T.J., BLACK K.S., PATERSON D.M., MITCHENER H.J., TERMAAT G.R., SHAYLER S.A. (2000). A comparison and measurement standardisation of four in situ devices for determining the erosion shear stress of intertidal sediments. Continental Shelf Research, 20(10-11), pp 1397-1418. doi:10.1016/S0278-4343(00)00029-7

UHLHERR P.H.T., GUO J., TIU C., ZHANG X.M., ZHOU J.Z.Q., FANG T.N. (2005). The shear-induced solid-liquid transition in yield stress materials with chemically different structures. Journal of Non-Newtonian Fluid Mechanics, 125(2-3), pp 101-119. doi:10.1016/j.jnnfm.2004.09.009

WINTERWERP J.C., VAN KESTEREN W.G.M. (2004). Introduction to the physics of cohesive sediment dynamics in the marine environment. Elsevier, $576 \mathrm{p}$. 